\title{
Vinorelbine as third line therapy in patients with advanced non-small cell lung cancer: Department of Clinical Oncology - Ain Shams University experience
}

\author{
Nesreen Mosalam¹, Engi El Kholy ${ }^{1}$, Mai Ezz EI Din ${ }^{1}$ \\ ${ }^{1}$ Department of Clinical Oncology, Faculty of Medicine, Ain Shams University.
}

Background: Lung cancer is the leading cause of cancer deaths. The treatment of advanced non small cell lung cancer (NSCLC), which carries a dismal prognosis, remains a challenging task. Our aim was to describe a single institution experience in using Vinorelbine single agent as salvage therapy (third line) in patients with locally advanced or metastatic NSCLC.

Patients and Methods: Data was collected from 2011 till 2014. Patients with locally advanced or metastatic NSCLC were enrolled in this study provided that these patients already received platinum based first line chemotherapy then second line docetaxel with good performance status. Twenty five patients were eligible. One patient lost follow up and two patients stopped treatment due to toxicity. All patients received Vinorelbine $25 \mathrm{mg} / \mathrm{m}^{2}$ on days 1,8 and 15 every 28 days. Patients were evaluated for response after 2 cycles. Responding patients and those with stable disease received maximum of six cycles. Primary end point was response rate. Secondary end points included safety, overall survival and time to progression.

Results: Of the eligible patients $18(72 \%)$ were males and $7(28 \%)$ were females. Eighteen (72\%) patients were aged between 30 and 65 years old. Nine (36\%) patients had stage IIIB and $16(64 \%)$ patients had stage IV. The ECOG performance status was 1 in $13(52 \%)$ patients and 2 in 12 (48\%). Objective response was seen in $13(52 \%)$ patients, $2(8 \%)$ patients had partial response and $11(44 \%)$ patients had stable disease. No complete response was reported and $9(36 \%)$ patients showed progressive disease. Median number of cycles of Vinorelbine was 5. Non hematological toxicity was well manageable mainly neuropathy, fatigue, constipation and hepatic toxicity. Ten $(40 \%)$ patients experienced hematological toxicity in the form of neutropenia and anemia. Seven patients (28\%) developed local toxicity at the injection site but none were grade 3 or 4 local toxicity .The median PFS was 4 months and the median overall survival was 10 months.

Conclusion: Generally Vinorelbine single agent as third line therapy for NSCLC is active and well tolerated.

Key words: NSCLC, Vinorelbine, third line therapy.

Corresponding Author: Nesreen Mosalam

E-mail: dr.nesreen2000@gmail.com

\section{INTRODUCTION}

Non-small cell lung cancer (NSCLC) represents 80$85 \%$ of all lung cancer cases, accounts for approximately $1213 \%$ - of all new cases annually and $2631 \%$ - of annual cancer related deaths in the United States ${ }^{1}$.

Despite recent and continued advances in surgical techniques, radiation and systemic treatment; The relative 5 years survival rate of a newly diagnosed patients with NSCLC is $15 \%$ with only modest improvement over the past 80 years $^{1,2}$.

Patients with NSCLC typically present with symptomatic and advanced disease because of this, together with relatively high rate of recurrence for earlier stage lesions. Palliative systemic therapy functions as corner stone for treatment of most cases ${ }^{3}$.

In advanced or recurrent NSCLC, a benefit to first line platinum-based chemotherapy doublet has been consistently demonstrated ${ }^{4}$.
Food and drug administration had approved docetaxel, pemetrexed and erlotinib for the treatment of advanced NSCLC in the second line setting 5 .

Chemotherapy beyond second line for advanced NSCLC is traditionally considered to have very little impact on overall survival ${ }^{6}$.

Vinorelbine is one of the chemotherapeutic agents with broad spectrum activity in epithelial malignancies. It arrests mitosis and induces apoptosis by inhibiting tubuline assembly. This agent has demonstrated activity in NSCLC, breast and ovarian cancers. It has been evaluated in NSCLC in the adjuvant and metastatic settings as single agent or in combination with other agents as platinum or gemcitabine with modest success ${ }^{7,8}$.

Despite reported activity in small studies, $, 9,10$. other studies evaluating vinorelbine as a single agent in the salvage setting had yield rather poor results ${ }^{11}$. 


Vol. 10|No. 3-4 $2014 \quad$ Nesreen Mosalam et al

The purpose of this study is to define the potential benefit of salvage vinorelbine in terms of efficacy and safety in heavily pretreated patients with advanced or metastatic NSCLC at Clinical Oncology Department Ain Shams University.

\section{PATIENTS AND METHODS}

\section{Selection of patients}

Between February 2011 and February 2014 patients with histologically proven stage IIIb or stage IV NSCLC were enrolled in the study who were previously treated with platinum based chemotherapy and docetaxel chemotherapy, Eastern Cooperative Oncology Group performance status (ECOG) less or equal to 2, adequate bone marrow function(white blood cell count of 4.000$11.000 / \mathrm{mm}^{3}$, neutrophil count of $2.000 / \mathrm{mm}^{3}$ or more, platelet count of $100.000 / \mathrm{mm}^{3}$ or more and hemoglobin level of $9.0 \mathrm{~g} / \mathrm{dl}$ or more), renal function (serum creatinine levels less than $1.5 \mathrm{mg} / \mathrm{dl}$ ), hepatic function (total serum bilirubin level within the upper limits of the normal range, levels of aspartate aminotransferase and alanine aminotransferase less than or equal to $2 \mathrm{x} U L N$ ).

Exclusion criteria included: presence of brain metastasis not receiving radiotherapy, ECOG performance status $>2$, severe or uncontrolled cardiac disease, severe or uncontrolled peripheral neuropathy, previous treatment with vinorelbine chemotherapy, pregnancy (for female patients), presence of other malignancy.

\section{Pretreatment evaluation}

Before treatment all eligible patients underwent complete medical history and physical examination, chest. Laboratory tests included a complete blood picture with differential count, liver function and renal function tests. Radiological investigations included pelvi-abdominal computed tomography scan, brain magnetic resonance imaging and bone scan.

\section{Treatment plan}

Eligible patients received vinorelbine as a salvage single agent in a dose of $25 \mathrm{mg} / \mathrm{m}^{2}$ on days 1,8 and 15 every 28 days. Patients were evaluated every 2 cycles and vinorelbine was continued for a maximum of 6 cycles for patients who had objective response (complete, partial response or stable disease ) as defined by Response Evaluation Criteria in Solid Tumors (RECIST criteria) ${ }^{12}$. Patients who developed progressive disease were excluded from this study.
Evaluation of toxicity was done every 2 cycles of treatment according to Common Toxicity Criteria (CTC) version ${ }^{4}$.

\section{RESULTS}

The study included 25 patients with histological diagnosis of NSCLC, one patient lost to follow up and 2 patients stopped treatment due to toxicity.

The characteristics of patients are shown in Table 1 .

Their median age was 54 years and the majority (72\%) was males. Almost half $(52 \%)$ had adenocarcinoma and two thirds (64\%) had a metastatic disease. The outcome of treatment is shown in Table 2. Treatment-related toxicities are summarized in Table 3. Seven $(28 \%)$ patients had local toxicity in the form of dermatitis at the site of injection but none had grade III or IV local toxicity.

Regarding hematological toxicity; 10 (40\%) patients developed grade I ,II anemia and neutropenia, 1 patient had Grade III anemia and 1 patient developed Grade IV neutropenia which required hospitalization, isolation and GCSF treatment and stopped treatment (table 3) .

Non-Hematological toxicities included neuropathy, fatigue, hepatic toxicity, constipation and alopecia. Neuropathy occurred in six $(24 \%)$ patients, 4 were Grade II and 1 was Grade III, 1 was Grade IV and stopped treatment protocol. Four (16\%) patients experienced fatigue Grade II, 1 (4\%) patient experienced hepatic toxicity grade II, $3(12 \%)$ patients experienced constipation Grade II. Alopecia did not occur with this protocol and nausea, vomiting were uncommon.

The mean number of treatment cycles was 4.4 and the median was 5 (range: 1 -6).

The mean overall survival (OS) was 18.5 months and the median was 10 months (Figure. 1).

The mean progression free survival (PFS) was 3.7 months and the median was 4 months (Figure. 2).

There was a statistically significant relation between response and mean number of cycles $(p=0.026)$. The mean number of cycles was 5.5 $( \pm 0.7)$ in patients with partial response, $4.9( \pm 1)$ in patients with stable disease and $3.8( \pm 1.4)$ in patients with disease progression. 
Survival Function

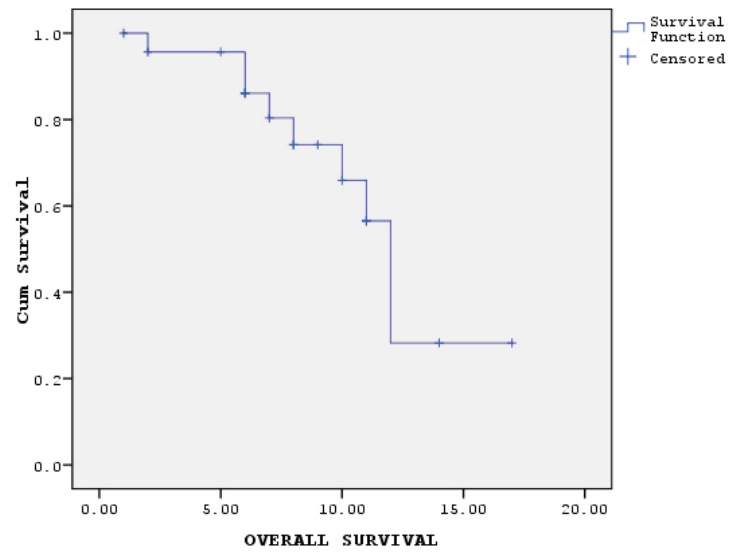

Figure 1: Overall survival curve (Kaplan Meier).

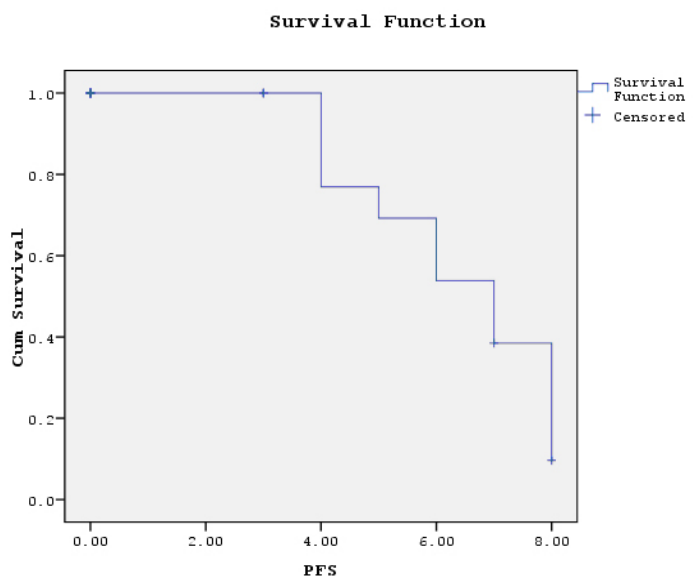

Figure. 2: Progression free survival curve (Kaplan Meier).

Table 1: Patients' characteristics.

\begin{tabular}{|c|c|c|c|}
\hline & & No. & $\%$ \\
\hline \multirow{2}{*}{ Gender } & Male & 18 & 72 \\
\hline & Female & 7 & 28 \\
\hline \multirow{2}{*}{ Age } & $31-65$ years & 18 & 72 \\
\hline & $>65$ years & 7 & 28 \\
\hline \multirow{3}{*}{ Pathology } & Squamous cell carcinoma & 4 & 16 \\
\hline & Adenocarcinoma & 13 & 52 \\
\hline & large cell carcinoma & 8 & 32 \\
\hline \multirow{2}{*}{ Presentation } & Locally advanced (IIIb) & 9 & 36 \\
\hline & Metastatic (IV) & 16 & 64 \\
\hline \multirow{2}{*}{ Performance status } & 1 & 13 & 52 \\
\hline & 2 & 12 & 48 \\
\hline
\end{tabular}




Vol. 10 | No. 3-4 $2014 \quad$ Nesreen Mosalam et al

Table 2: Treatment outcome.

\begin{tabular}{lll}
\hline & N. & \% \\
\cline { 2 - 3 } Complete remission & 0 & 0 \\
\hline Partial response & 2 & 8 \\
\hline Stable disease & 11 & 44 \\
\hline Disease progression & 9 & 36 \\
\hline Stopped due to toxicity & 2 & 8 \\
\hline Lost to follow up & 1 & 4 \\
\hline
\end{tabular}

Table 3: Treatment-related toxicities.

\begin{tabular}{lll}
\hline Toxicity & $\boldsymbol{n}$ & $\mathbf{\%}$ \\
\hline Local & 7 & 28 \\
\hline Hematological & 10 & 40 \\
\hline Non-hematological & & \\
\hline Neuropathy & 6 & 24 \\
\hline Fatigue & 4 & 16 \\
\hline Constipation & 3 & 12 \\
\hline Hepatic toxicity & 1 & 4 \\
\hline
\end{tabular}

\section{DISCUSSION}

In our study vinorelbine single agent demonstrated activity as a salvage therapy for patients with locally advanced or metastatic NSCLC. Two patients $(8 \%)$ had partial response, 11 (44\%) had stable disease and 9 $(36 \%)$ had progressive disease. None of our patients had complete response. The median number of cycles was 5, median PFS was 4 months and the median OS was 10 months. Our results were comparable to that reported by Subramanian et $\mathrm{al}^{14}$ as $3 \%$ of patients had partial response, 13 patients $(38 \%)$ had stable disease and 16 patients $(47 \%)$ had progressive disease. Similarly, none of their patients had complete response. The median PFS was 5.6 months and the median OS was 10.7 months ${ }^{14}$.

Likewise our results are comparable to that reported by Devlin and Langer who reported $7.7 \%$ partial response, $25.6 \%$ stable disease and $43.6 \%$ progressive disease with a median number of cycles of $3^{3}$. The median time to progression was 3 months and the median time to progression after excluding patients with progressive disease and patients not assessed for response was 8 months. They reported a lower median OS of 5 months but in our study was 10 months. This can be explained by their inclusion of patients with performance status more than 2 in their study ${ }^{3}$.

Regarding toxicity the most commonly seen toxicity was hematological toxicity in 10 patients $(40 \%)$ in the form of anemia and neutropenia. Hematological toxicities were mostly mild to moderate cases except one patient who developed grade IV neutropenia needing hospitalization and treatment discontinuation afterward. Other non-hematological toxicities were reported in the form of neuropathy in $(24 \%)$ of the patients, all of which were well controlled except one patient who developed grade IV neuropathy and treatment was discontinued. Sixteen percent had fatigue, $4 \%$ had hepatic toxicity and $12 \%$ had constipation. These results were comparable to that reported by Hainsworth et al. ${ }^{15}$ who reported predominance of hematological toxicities. In their study one patient had grade IV leukopenia and 5 patients had grade IV granulocytopenia. Regarding the nonhematologic toxicities, fatigue was reported in 9 patients, nausea and vomiting in 2 patients and none had grade IV toxicity. Our results were comparable to those reported by Devlin and Langer who reported predominance of hematological toxicities, 3 patients had grade III, 3 patients had grade IV neutropenia. ${ }^{3}$ These rates were higher than ours. This may be due to the different chemotherapy schedule used in that study $\left(30 \mathrm{mg} / \mathrm{m}^{2}\right.$ on days 1 and 8 every 21 day) $)^{3}$. One patient had grade III anemia. Regarding the non-hematologic toxicities they were similar to our study, they reported mild to moderate fatigue in 3 patients, neuropathy grade III in one patient and grade III gastrointestinal upset in one patient ${ }^{3}$.

\section{CONCLUSION}

The prognosis of patients with advanced NSCLC is poor. Systemic treatment remains the mainstay of palliation and to improve symptoms, QOL and survival. Vinorelbine remains a safe and effective systemic therapy for patients with advanced NSCLC, even as third line. Salvage vinorelbine may be considered a reasonable treatment option.

\section{REFERENCES}

1. Jemal A, Siegel R, Ward et al. Cancer statistics. CA Cancer J Clin. 2006;56:106-130.

2. Parkin D, Gray H and Devessa S. Cancer burden for the year 2000. The global picture. Eur J Cancer. 2001;37:S4-S66.

3. Devlin JG and Langer CJ. Salvage therapy with Vinorelbin in advanced non small cell lung cancer, Clin Lung Cancer. 2007;8:319-326.

4. Non-small cell lung cancer Collaborative Group (1995): Chemotherapy in non small cell lung cancer . a metaanalysis updated data on individual patients from 52 randomized clinical trials. B M J ;311:899-909.

5. Ho C, Davies AM, Lara PN, et al. Second-line treatment for advanced-stage non-small-cell lung cancer: current and future options. Clin Lung Cancer. 2006; 7:S118-S125. 
6. Noble J, Ellis PM, Mackay JA, et al. (2006): second - line or subsequent systemic therapy for recurrent or progressive non small cell lung cancer. a systematic review and practical guidelines. $\mathrm{J}$ Thorac Oncol. 2006;1:1042-1058.

7. Fossella F, Devore R, Kerr R, et al. Randomized phase III trial of Docetaxel versus Vinorelbine or Ifosfamide in patients with advanced non small cell lung cancer previously treated with Platinum containing chemotherapy regimens. J Clin Oncol. 2000;18:23542362.

8. Gridelli C and De Vivo.R. Vinorelbine in the treatment of non small cell lung cancer. Curr Med Chem. 2002;9:879-891.

9. Miyata J, Sano T and Bandu H. [A trial with single agent Vinorelbine in Taxaenes resistant non small cell lung cancer]. Gan To Kagalu Ryoho. 2001;28:239-242. [Article in Japanese]

10. Miyata J, Sano T and Bandu H. Effect of Vinorelbine monotherapy in Taxenes treated patients with advanced non small cell lung cancer. Med Oncol. 2002;19:11-14.
11. Douillard JY, Gervais R, Dobouis G, et al.Sequential two lines strategy for stage IV non small cell lung cancer .Docetaxel-Cisplatin versus Vinorelbine - Cisplatin followed by cross over to single agent Docetaxel or Vinorelbine at progression :final results of a randomized phase II study. Ann Oncol. 2005;16:81-89.

12. Therasse A, Arbuck S, Eisenhauer E.,et al. New guidelines to evaluate the response to treatment in solid tumors .J Natl Cancer Inst. 2000;92;205-216.

13. Kaplan ZL and Meier P. Non parametric estimation from incomplete observation. J Am Stat Assoc. 1958;53:457-81.

14. Subramanian J, Velcheti V, Baggstrom MQ, et al. Efficacy of Vinrolbine in the second line setting and beyond in non small cell lung cancer. J Thorac Oncol. 2007;2:373.

15. Hainsworth J, Burris H, Litchy S, et al. Gemcitabine and Vinrolbine in the second line treatment of non small cell lung carcinoma patients. Cancer. 2000;88;1353-1358. 